\title{
Sources and Types of Discrepancies Between Electronic Medical Records and Actual Outpatient Medication Use
}

\author{
Kathleen B. Orrico, PharmD, BCPS
}

\begin{abstract}
BACKGROUND: Accuracy and transportability of the recorded outpatient medication list are important in the continuum of patient care. Classifying discrepancies between the electronic medical record (EMR) and actual drug use by the root cause of discrepancy (either system generated or patient generated) would guide quality improvement initiatives.
\end{abstract}

OBJECTIVES: To quantify and categorize the number and type of medication discrepancies that exist between the medication lists recorded in EMRs and the comprehensive medication histories obtained through telephone interviews conducted by a team of nurses providing advice to health plan members at the Palo Alto Medical Foundation in Palo Alto, California.

METHODS: The study was conducted as a retrospective comparison of EMR medication lists with information obtained by patient interview. Interview data were obtained by a review of telephone calls made to a nurse advice line by health plan members seeking information about sinusitis, urinary tract infection, acute conjunctivitis, pharyngitis, emergency contraception, or mastitis. As part of the advice protocol, a medication reconciliation process was conducted between July 1 and December 31, 2006. Changes to the medication list made during the telephone visit were extracted, categorized, and evaluated by the study's principal investigator. Data extraction included the number and type of identified medication discrepancies, patient age, gender, and condition that prompted the telephone contact. A modified version of the Medication Discrepancy Tool (MDT) was used to categorize medication discrepancies as either system generated (e.g., failure of the provider to update a medication list) or patient generated (e.g., failure of the patient to report use of an over-the-counter product).

RESULTS: A total of 233 discrepancies were identified from 85 medication reconciliation phone visits, averaging 2.7 per medication list. The most common type of discrepancy was a medication recorded in the EMR but no longer being used by the patient $(n=164,70.4 \%)$, followed by omission from the EMR of a medication being taken by the patient $(n=36,15.5 \%)$. $79.8 \%(n=186)$ of the discrepancies were attributed to system-generated factors, whereas $20.2 \%(n=47)$ were patient generated. Approximately half $(n=118,50.6 \%)$ of the discrepancies fell into 4 broad American Hospital Formulary System therapeutic classifications: anti-infective agents (14.2\%), anti-inflammatory agents $(14.2 \%)$, analgesics $(12.4 \%)$, and vitamins $(9.9 \%)$. The most common patient-generated discrepancy was omission of a multivitamin ( $n=13,27.7 \%)$, and the most common systemgenerated prescription drug discrepancy was expired entry for the intranasal corticosteroid mometasone furoate $(n=12,6.5 \%)$.
CONCLUSION: Discrepancies in the outpatient setting were common and predominantly system generated. The most common discrepancy was the presence in the EMR of a medication no longer being taken by the patient. Adding foreseeable end dates to prescription drug orders at computerized order entry might be considered in an effort to improve the accuracy of the outpatient medication list. Reliable systems to involve patients in routinely reconciling EMRs with actual medication use may also warrant examination. The MDT methodology served as a useful qualitative guide for evaluating discrepancies and developing targeted means for resolution.

J Manag Care Pharm. 2008;14(7):626-631

Copyright $\odot$ 2008, Academy of Managed Care Pharmacy. All rights reserved

\section{What is already known about this subject}

- At hospital admission, an estimated $60 \%-67 \%$ of medication histories contain at least 1 error, either omission of a medication being taken, or reporting of a medication not being used. An estimated 11\%-59\% of these errors are clinically important.

- A comparison of hospital discharge orders and a comprehensive medication assessment conducted after discharge identified medication discrepancies in $14.1 \%$ of patients aged 65 years and older and with at least 1 chronic condition. Of these discrepancies, $50.8 \%$ were patient generated and $49.2 \%$ were system generated.

\section{What this study adds}

- For 85 outpatients contacting a health plan medical advice line for assistance with relatively minor medical conditions, a total of 407 medication entries in an electronic medical record (EMR) were identified. Of those, 233 (57.2\%) did not match to information obtained using a comprehensive medication assessment conducted telephonically by a nurse.

- In the majority (70.4\%) of discrepancies between EMR entries and medication assessments, a medication recorded in the EMR was no longer being taken by the patient.

- $79.8 \%$ of the discrepancies were system generated, whereas 20.2\% were patient generated. 


\section{Sources and Types of Discrepancies Between Electronic \\ Medical Records and Actual Outpatient Medication Use}

$\mathrm{M}$ any studies evaluating medication reconciliation have focused on activities occurring at times of transition during or immediately following hospital care..$^{1-6}$ Cornish et al. reported that upon hospital admission, few patients could produce an accurate account of their current medication regimens, and that the most common error identified in the medication use history was the omission of a regularly used medication (46.4\%). ${ }^{1}$ Because the genesis of most prescription drug orders occurs in the physician office setting, improving the accuracy and transportability of the recorded outpatient medication list would extend a measure of quality throughout the continuum of patient care.

People are particularly vulnerable for experiencing medication mishaps during care transitions such as hospital discharge. Coleman et al.'s study of 375 elderly patients assessed 1 week post-hospitalization showed that $14.1 \%$ had experienced 1 or more medication discrepancies, defined as a lack of agreement between prescribed drug therapy indicated on the hospital discharge record and the therapy actually received by the patient. ${ }^{2}$ The study used the Medication Discrepancy Tool (MDT), a methodology developed and tested for reliability by Smith et.al, to categorize these transition-related discrepancies as being generated at either the system level (i.e., under the control of providers) or the patient level (i.e., under the control of patients). ${ }^{3}$ Of the 124 discrepancies identified in the Coleman et al. study, $49.2 \%$ were categorized as system generated and $50.8 \%$ were categorized as patient generated. ${ }^{2}$ A systematic review of studies of medication errors at hospital admission indicated that $60 \%-67 \%$ of prescription medication histories contained at least 1 error, either the omission of a medication being taken by the patient or the reporting of a medication not being taken. An estimated 11\%-59\% of these errors were deemed clinically important. ${ }^{6}$

The objective of this study was to determine the state of accuracy of the outpatient medication list by quantifying and categorizing medication discrepancies between the recorded medication list contained in the EMR and a comprehensive medication history obtained through telephone interview by an advice nurse at the Palo Alto Medical Clinic (PAMC). The intended use of a modified MDT to classify the root cause of each discrepancy as either system or patient generated was to allow quality improvement activities to be appropriately devised and directed.

\section{Methods}

\section{Study Setting and Patient Population}

The study was conducted at the PAMC, a division of the Palo Alto Medical Foundation for Health Care, Research, and Education located in Northern California, and was approved by the Palo Alto Medical Foundation Institutional Review Board. The foundation is an affiliate of Sutter Health, a system of not-for-profit hospitals and physician organizations. PAMC, a multispecialty medical group with approximately 250 physicians and 900 other professionals at its main facility and 4 satellite clinics, is respon- sible for approximately 190,000 covered enrollees.

In 2002, PAMC implemented a full-featured EMR, allowing electronic documentation of all clinician activity, including computerized medication order entry and the generation of a medication list. Because prescription order entry is a universally used feature, the medication list contains all prescription orders generated at PAMC, including scheduled medications (i.e., controlled substances). Prescription orders are entered into the system and sent by fax to any retail pharmacy. The physician also can generate printed copies of prescriptions, including scheduled medications, which are routed to secure printers containing tamper-proof forms as required by California law. The system allows for entry of over-the-counter (OTC) items, herbal medications, and nutraceuticals. As a standard practice at PAMC, medications prescribed by providers outside of PAMC but reported as being actively received by the patient are recorded on the medication list and given the system designation of "historical medication," indicating that the information is patient reported.

The study was conducted as a retrospective, encounter-based EMR review of telephone calls made to a nurse advice line in the PAMC Department of Family Medicine from July through December 2006. Registered nurses are authorized to enact standardized procedures and telephone treatment protocols for the following complaints: acute conjunctivitis, emergency contraception, mastitis in breast feeding women, pharyngitis, sinusitis, and urinary tract infection in women. To qualify to enact these protocols, a nurse must have at least 2 years of prior experience as a registered nurse, have a current California nursing license, and have passed an initial and annual competency evaluation. Only patients who received their primary care at PAMC and who met specific symptom criteria for 1 of the 6 conditions listed above were eligible for telephone treatment and received medication reconciliation. A sample was identified for review by randomly selecting 10 clinic log sheets from approximately 150 clinic log sheets recorded during the 6-month data collection time period. Each log sheet listed the date of service and medical record number of the patient receiving medication reconciliation. No other restrictions were placed on the group of patients included in the study.

Beginning in July 2006, the 8 advice-line nurses began to incorporate a medication reconciliation process into their usual workflow. During the telephone visit (encounter), patients were asked to verify their current medication regimen and to include medications prescribed by physicians outside of PAMC, OTC medications, and herbal preparations. The nurse first verified the medication list by naming each recorded medication using both the generic and brand name, if applicable, and then asking the patients whether they were actively taking that medication. The dose, frequency, and route of administration were also verified with the patient. For medications prescribed on an as-needed basis, such as medications for pain or allergies, patients were asked whether they had used the drug within the past year. The 


\section{Sources and Types of Discrepancies Between Electronic \\ Medical Records and Actual Outpatient Medication Use}

medication was left on the medication list if the patient reported use within the past year or if the patient requested that it remain listed. Next, the patient was asked to report any active use of OTC items, herbal supplements, nutraceuticals, vitamins, and any other prescription medication not recorded on the medication list, including medications prescribed by providers not affiliated with PAMC. At the end of the interview, the nurse updated the medication list. Electronic notification was sent to the primary care physician for review and approval of all changes made including additions and deletions.

\section{Medication Discrepancy Classification}

Changes and updates to the medication list enacted during the 85 telephone visits were extracted, quantified, and classified using a retrospective comparison with the EMR. Data extraction was conducted by the principal investigator, and each alteration made to the medication list during the nurse visit was transferred to Microsoft Excel worksheets for categorization. The medical record number was the only patient identifier extracted. Additional data points extracted included patient age, sex, date of visit, and which of the 6 medical conditions prompted the call to the advice line.

For the purpose of this evaluation a "discrepancy" was defined as any lack of agreement between the medication list in the EMR and the patient-reported medication regimen. Discrepancies included any incongruity in medication, dose, frequency, or route, as well as any omission, duplication of the same medication in the EMR list, or drug no longer being taken by the patient remaining current on the EMR list. Each discrepancy was assigned to a therapeutic classification based on the American Hospital Formulary Service (AHFS) standard. ${ }^{7}$ The AHFS therapeutic classification categories are extremely broad and can include oral, ophthalmic, topical, nasal, inhaled, and oral agents. For example, the classification of anti-inflammatory drugs would include oral methylprednisolone tablets, montelukast tablets, mometasone nasal spray, fluticasone inhalation, desonide topical, and hydrocortisone suppositories.

Discrepancies also were assigned to categories based on the MDT methodology developed by Smith et al. ${ }^{3}$ The MDT was created to identify health care facility transition-related medication discrepancies and assign them to actionable categories. A main element of the MDT is the classification of each discrepancy as being either system generated or patient generated. This initial assignment allows for the analysis of the root cause of a discrepancy. A system-generated discrepancy occurred if the physician or a health system process was deemed to have control or responsibility for the medication list inaccuracy. An example of a system-generated discrepancy in the outpatient record was the current listing on the medication list of an antibiotic, such as ciprofloxacin for the treatment of a urinary tract infection, long after the course of therapy had been completed. A discrepancy was classified as patient generated if it occurred because of a fac- tor under the patient's control. An example of a patient-generated discrepancy was the omission from the medication list of a daily multivitamin that the patient initiated; this discrepancy is considered patient generated, because the only means for inclusion of this product in the EMR is through patient report.

In accordance with the MDT methodology, discrepancies were further classified into subcategories based on contributing factors. The intent of this action is to explore the frequency, causes, and contributing factors to the generation of medication discrepancies. Adaptations of the original tool were made to make the subcategorization more applicable to the study. For example, MDT subcategories such as "Did not fill prescription" and "No caregiver" did not apply to this study setting. For the purpose of this study, subcategories included duplicate entry of the same generic entity, omission from the EMR of a medication that the patient reported receiving, erroneous entry of either the dose or directions, and inclusion on the EMR of a medication that the patient reported no longer receiving.

To identify causal factors and provide guidance for corrective action planning, a third categorization scheme that was not part of the original MDT was added to the analysis. Each systemgenerated discrepancy was categorized as caused by (a) an end date not entered at the time of prescribing when it was foreseeable to determine length of therapy or (b) failure to update the medication list. Patient-generated discrepancies were categorized as caused by (a) OTC medication/product being taken by the patient but not listed in the EMR, (b) prescription medication prescribed by a non-PAMC provider, or (c) reported intentional nonadherence. Discrepancies involving OTC medications and those prescribed by non-PAMC physicians (historical medications) are classified as patient generated because the patient has firsthand knowledge of current use; thus, strategies to resolve the discrepancy depend on updates provided by the patient.

\section{Results}

\section{Population Characteristics}

The 85 evaluated medication reconciliation telephone calls were associated with 85 unique patients who were all assigned to a primary care provider at PAMC. Because the study included only patients who met specific symptom criteria for 1 of the 6 conditions managed by telephone treatment protocol, the study population was skewed towards women (female $n=69$ [81.2\%]), with a mean (SD) age of 42 (14) years (Table 1). Medical conditions reported by callers included: sinusitis $(n=38,44.7 \%)$, urinary tract infection in adult women $(\mathrm{n}=26,30.6 \%)$, acute conjunctivitis $(n=9,10.6 \%)$, pharyngitis $(n=7,8.2 \%)$, emergency contraception $(n=4,4.7 \%)$, and mastitis in breastfeeding women $(n=1,1.2 \%)$.

\section{Types of Medication Discrepancies}

The 85 recorded medication lists contained a total of 407 current medication entries, an average of 4.8 entries per list. A total of 233 discrepancies were identified (average of 2.7 per list) and cat- 


\section{Sources and Types of Discrepancies Between Electronic \\ Medical Records and Actual Outpatient Medication Use}

egorized as resulting from duplicate entries ( $\mathrm{n}=27,11.6 \%$ ), active medications omitted from the profile $(n=36,15.5 \%)$, medications recorded in the EMR but no longer being taken ( $\mathrm{n}=164,70.4 \%)$, and differences in dosage or directions $(\mathrm{n}=6,2.6 \%$; Table 2).

Each discrepancy was further classified as being either system-generated $(n=186,79.8 \%)$ or patient-generated $(n=47$, $20.2 \%$; Table 2). The majority ( $\mathrm{n}=155,83.3 \%)$ of the 186 systemgenerated discrepancies resulted from discontinued or expired medications that had been left active on the medication list. The most frequently occurring patient-generated discrepancies ( $\mathrm{n}=36$, $76.6 \%$ ) resulted from omission from the EMR of OTC medications and those prescribed by providers outside of PAMC.

Categorization of discrepancies based on causal factors is summarized in Table 3. An actionable causation factor for approximately half $(48.4 \%)$ of the system-generated discrepancies was the lack of the entry of an end date for medications with known lengths of therapy. A causal factor for more than half (61.7\%) of the patient-generated discrepancies was the lack of documentation for active OTC medication or product use.

\section{Medication Classes}

Half of the 233 identified discrepancies ( $\mathrm{n}=118,50.6 \%$ ) fell into 4 broad AHFS medication classes: anti-infective agents (14.2\%), anti-inflammatory agents (14.2\%), analgesics (12.4\%), and vitamins (9.9\%; Table 4). The first 3 categories contained medications that typically have a finite or seasonal course of use (antibiotics, analgesics, nasal corticosteroids). The omission of a multivitamin from the EMR list was the most commonly occurring patientgenerated discrepancy ( $n=13,27.7 \%$, data not shown). An entry for the intranasal corticosteroid mometasone furoate, a seasonal anti-inflammatory agent, was the prescription drug most commonly occurring as a system-generated discrepancy because it remained on the medication list after active use had stopped ( $n=12,6.5 \%$, data not shown).

\section{TABLE 1 Characteristics of Outpatients Telephoning} a Health Plan Nurse Advice Line

\begin{tabular}{l|c|}
\hline Number of unique patients & 85 \\
\hline Age (years) mean (SD) & $42(14)$ \\
\hline Variable & $\mathbf{N}(\%)$ \\
\hline Age range (years), by age category & \\
\hline $18-89$ & $34(40.0)$ \\
\hline $18-39$ & $45(52.9)$ \\
\hline $40-59$ & $6(7.1)$ \\
\hline $60-89$ & $16(18.8)$ \\
\hline Male & $69(81.2)$ \\
\hline Female & $38(44.7)$ \\
\hline Medical Condition Reported by Patient & $26(30.6)$ \\
\hline Sinusitis & $9(10.6)$ \\
\hline Urinary tract infection in women & $7(8.2)$ \\
\hline Acute conjunctivitis & $4(4.7)$ \\
\hline Pharyngitis & $1(1.2)$ \\
\hline Emergency contraception & \\
\hline Mastitis in breastfeeding women & \\
\hline
\end{tabular}

\section{Discussion}

The results of the study showed that the EMR medication list contained many discrepancies, the majority of which were attributable to system-generated factors. True inaccuracies, such as the entry into the EMR of an incorrect dose or frequency or the entry of a duplicate order entry, were rare. Because the majority of discrepancies were the result of discontinued medications being allowed to remain on the list long after the course of therapy had been completed, it follows that discrepancies fell into therapeutic classifications that have a finite or seasonal, rather than chronic, treatment course.

\section{TABLE 2 Categorization of Medication Discrepancies by System-Generated and Patient-Generated Factors}

\begin{tabular}{|c|c|c|c|c|}
\hline Factors & Definition & $\begin{array}{c}\text { System Generated } \\
\text { n (\%) }\end{array}$ & $\begin{array}{c}\text { Patient Generated } \\
\mathrm{n}(\%)\end{array}$ & $\begin{array}{l}\text { Total } \\
\text { n }(\%)\end{array}$ \\
\hline & & $186(79.8)$ & $47(20.2)$ & $233(100)$ \\
\hline Duplicate entry & Generic entity listed more than once & $27(14.5)$ & 0 & $27(11.6)$ \\
\hline Omission from EMR & $\begin{array}{l}\text { Patient reports currently receiving a medi- } \\
\text { cation that is not listed }\end{array}$ & 0 & $36(76.6)$ & $36(15.5)$ \\
\hline Patient not receiving_current on EMR & $\begin{array}{l}\text { Medication present on EMR, but patient } \\
\text { reports not currently receiving }\end{array}$ & $155(83.3)$ & $9(19.1)$ & $164(70.4)$ \\
\hline Erroneous entry-dose & $\begin{array}{l}\text { Medication entry on EMR shows different } \\
\text { dose than what patient reports receiving }\end{array}$ & $3(1.6)$ & $1 \quad(2.1)$ & $4 \quad(1.7)$ \\
\hline Erroneous entry-directions & $\begin{array}{l}\text { Medication entry on EMR shows differ- } \\
\text { ent directions than what patient reports } \\
\text { receiving }\end{array}$ & $1 \quad(0.5)$ & $1 \quad(2.1)$ & $2(0.9)$ \\
\hline
\end{tabular}




\begin{tabular}{|c|c|}
\hline \multicolumn{2}{|l|}{ System-Generated Factors } \\
\hline$n=186$ & n $\quad(\%)$ \\
\hline Medication list not updated & $96(51.6)$ \\
\hline End date not entered & $90(48.4)$ \\
\hline \multicolumn{2}{|l|}{ Patient-Generated Factors } \\
\hline$n=47$ & n $\quad(\%)$ \\
\hline Omission of OTC medication or product & $29(61.7)$ \\
\hline Prescribed by outside physician & $9(19.1)$ \\
\hline Intentional nonadherence & $9(19.1)$ \\
\hline
\end{tabular}

Although anti-infective and anti-inflammatory agents were the classes of medications found to be most often involved in discrepancies in this outpatient setting, medication discrepancies reported in previous studies conducted in hospital settings involved different categories of medications. Coleman et al.'s study of post-hospital discharge discrepancies found that anticoagulants (13\%), diuretics (10\%), angiotensin-converting enzyme inhibitors (10\%), and lipid-lowering agents (10\%), were the 4 most commonly occurring medication classes involved in discrepancies. ${ }^{2}$ Cornish et al.'s evaluation of discrepancies upon hospital admission found cardiovascular agents (26.6\%) and central

\section{TABLE 4 Top 10 Discrepancies Categorized by AHFS Therapeutic Class $^{a}$ ( $n=233$ )}

\begin{tabular}{|c|c|}
\hline Therapeutic Class & n $\quad(\%)$ \\
\hline Anti-infective agents ${ }^{\mathrm{b}}$ & $33(14.2)$ \\
\hline Anti-inflammatory agents ${ }^{c}$ & $33(14.2)$ \\
\hline Analgesics $^{\mathrm{d}}$ & $29(12.4)$ \\
\hline Vitamins & $23(9.9)$ \\
\hline Anxiolytics, sedatives, and hypnotics & $15(6.4)$ \\
\hline Contraceptives & $15(6.4)$ \\
\hline Antidepressants & $13 \quad(5.6)$ \\
\hline Beta-adrenergic agonists & $9(3.9)$ \\
\hline Supplements & $9(3.9)$ \\
\hline Antiemetic & $5(2.1)$ \\
\hline Total number of discrepancies in top 10 categories & $184(79.0)$ \\
\hline \multicolumn{2}{|c|}{$\begin{array}{l}\text { "Therapeutic categories are broad and include all dosage forms. } \\
{ }^{b} \text { Anti-infective agents include antibiotics, antifungals, and antiviral agents (e.g., azithro- } \\
\text { mycin tablets, ketoconazole tablets, and clindamycin topical). } \\
\text { 'Anti-inflammatory agents is a broad therapeutic category that includes oral, nasal, } \\
\text { topical, and rectal drugs (e.g., oral methylprednisolone tablets, montelukast tablets, } \\
\text { mometasone nasal spray, fluticasone inhalation, desonide topical, and hydrocortisone } \\
\text { suppositories). } \\
\text { dAnalgesics include nonsteroidal anti-inflammatory drugs, cyclooxygenase-2 inhibitors, } \\
\text { and opiate analgesics (e.g., ibuprofen tablets, celecoxib capsules, and acetaminophen, } \\
\text { codeine tablets). } \\
\text { AHFS=American Hospital Formulary Service }\end{array}$} \\
\hline
\end{tabular}

nervous system agents (25.9\%) to be most commonly identified ${ }^{1}$ The variation in identified medication classes may be the result of the differences in the populations studied. Both pre- and posthospitalization studies evaluated senior patients with high acuity, whereas our outpatient population represented a younger, less medically complicated group. A study that evaluated predictors of discrepancies in outpatient practice found through multivariate analysis that older age was strongly associated with the occurrence of medication discrepancies ${ }^{8}$ Results of the present study show that discrepancies are evident in younger age groups as well, suggesting the importance of process improvement activities directed at building reliable systems that affect the accuracy of medication lists for all patients.

Classifying the causes of patient-generated discrepancies was a useful approach and highlighted the need to partner with the patient in order to arrive at medication list accuracy. OTC products initiated by the patient accounted for $61.7 \%$ of the patientgenerated discrepancies. A prospective study of 104 primary care outpatients conducted at the Mayo Clinic, that evaluated the prevalence of medication discrepancies in the EMR, also found that omission of OTC drugs from the recorded medication list was a common discrepancy. ${ }^{9}$ In that study, a program of mailed letters reminding patients to bring medication information to appointments, coupled with verification and correction of medication lists, was associated with a decrease in discrepancies from $89 \%$ to $66 \%$ of visits. Phase I of the Mayo Clinic study compared the medication list recorded in the EMR with a reconciled medication list produced by a nurse telephone interview with the patient. Of the 147 OTC and herbal products reported as being taken by the patients, 87 (76\%) were omitted from the EMR medication list. ${ }^{9}$ Developing similar strategies for capturing this information from the patient would be valuable in addressing patient-generated discrepancies.

\section{Process Improvement}

The MDT methodology served as a useful qualitative guide for evaluating discrepancies and developing targeted means for resolution. The finding that the greatest number of system-generated discrepancies resulted from expired medications being left active on the medication lists permitted the development of a targeted, systematic corrective action plan. An examination of the top 3 AHFS therapeutic classes showed the presence of many medications for which the length of therapy is known at prescribing; the simple addition of an order end date to the EMR would automate removal from the EMR medication list.

To aid this effort, an educational campaign was launched at PAMC to encourage physicians to enter an appropriate end date to prescription orders when the length of therapy was predictable upon order entry. Chronic medications, which were rarely identified as discrepancies in our evaluation, were not to be given an end date. The information was presented to the Family Medicine physicians at a department meeting. As a process check, the 


\section{Sources and Types of Discrepancies Between Electronic \\ Medical Records and Actual Outpatient Medication Use}

percent of short-course azithromycin orders written with an end date was measured 30 days pre- and post-presentation. Before the presentation, 7 of 25 (28\%) total orders of azithromycin contained an end date. After the presentation the percentage rose to 16 of 23 (70\%). Plans are under way to automatically populate the end date field for many medications.

As part of its "5 Million Lives Campaign," the Institute for Healthcare Improvement recommends encouraging patients to play a major role in ensuring that the medication list is kept up to date as they visit multiple providers in the outpatient setting. ${ }^{10}$ Strategies for reducing patient-generated discrepancies have been explored by other medical groups.

Harvard Pilgrim Health Care conducts a comprehensive medication reconciliation program that involves direct pharmacist intervention with high-risk patients. ${ }^{11} \mathrm{~A}$ similar program that uses pharmacists to conduct a comprehensive medication reconciliation while a patient undergoes dialysis has been successful at the Dialysis Clinic Inc. in Kansas City, Missouri. ${ }^{12}$

\section{Limitations}

First, our study was limited to a small sample of outpatients who telephoned an advice-line nurse to request assistance with relatively minor medical conditions. Thus, our results may not be generalizable to a broader outpatient population. Second, the nurse interview process relied on patient self-report as a "gold standard" against which the EMR was compared. Errors made by patients in reporting drug, duration, or dose, or by nurses using the protocol could have overstated the rate of discrepancy between the EMR and the patient-reported information. Lastly, because data extraction was conducted by one person, errors or biases could have affected the data collection process.

\section{Conclusions}

Discrepancies in the outpatient setting were common and predominantly system generated. Establishing appropriate medication order end dates at the point of prescription order entry could eliminate $50 \%$ of the system-generated discrepancies in this study setting. Effective methods to improve medication list accuracy should address both system- and patient-generated discrepancies. A "stretch goal" for all outpatient office settings should be to supply every patient with an accurate list of active medications that would improve patient safety throughout the continuum of patient care. The MDT methodology served as a useful qualitative guide for evaluating discrepancies and developing targeted means for resolution.

\section{DISCLOSURES}

The author reported no funding for this study and no conflicts of interest related to the subject of this article.

\section{REFERENCES}

1. Cornish PL, Knowles SR, Marchesano R, et al. Unintended medication discrepancies at the time of hospital admission. Arch Intern Med. 2005; 165(4):424-29.

2. Coleman EA, Smith JD, Raha D, Min SJ. Posthospital medication discrepancies. Arch Intern Med. 2005;165(16):1842-47.

3. Smith JD, Coleman EA, Min SJ. A new tool for identifying discrepancies in postacute medications for community-dwelling older adults. Am J Geriatr Pharmacother. 2004;2(2):141-48

4. Kripalani S, LeFevre F, Phillips CO, Williams MV, Basaviah P, Baker DW Deficits in communication and information transfer between hospital-based and primary care physicians: implications for patient safety and continuity of care. JAMA. 2007; 297(8):831-41.

5. Kripalani S, Jackson AT, Schnipper JL, Coleman EA. Promoting effective transitions of care at hospital discharge: a review of key issues for hospitalists. J Hosp Med. 2007; 2(5):314-23.

6. Tam VC, Knowles SR, Cornish PL, Fine N, Marchesano R, Etchells EE. Frequency, type, and clinical importance of medication history errors at admission to hospital: a systematic review. CMAJ. 2005;173(5):510-15.

7. McEvoy GK, ed. AHFS Drug Information 2005. Bethesda, MD: American Society of Health-System Pharmacists; 2005.

8. Bedell SE, Jabbour S, Goldberg R, Glaser H, Gobble S, Young-Xu Y, et.al. Discrepancies in the use of medications. Arch Intern Med. 2000;160 (14):2129-34.

9. Varkey P, Cunningham J, Bisping S. Improving medication reconciliation in the outpatient setting. Jt Comm J Qual Patient Saf. 2007;33(5):286-92.

10. Institute of Medicine. Crossing the Quality Chasm: A New Health System for the 21st Century. Washington, DC: National Academy Press; 2001.

11. Bernstein L, Frampton J, Minkoff NB, et al. Medication reconciliation Harvard Pilgrim Health Care's approach to improving outpatient medication safety. J Healthc Qual.2007;29(4):40-45

12. Manley HJ, Drayer DK, McClaran M, Bender W, Muther RS. Drug record discrepancies in an outpatient electronic medical record: frequency, type, and potential impact on patient care at a hemodialysis center. Pharmacotherapy. 2003:23(2):231-39.

\section{Author}

KATHLEEN B. ORRICO, PharmD, BCPS, is Health Sciences Assistant Clinical Professor of Pharmacy, University of California, San Francisco, School of Pharmacy; and Clinical Pharmacist, Palo Alto Medical Foundation, Palo Alto, California.

AUTHOR CORRESPONDENCE: Kathleen B. Orrico, PharmD, BCPS, Palo Alto Medical Foundation, 795 El Camino Real, Pharmacy Level A, Palo Alto, CA 94301. Tel.: 650.614.3217; E-mail: orricok@pamf.org. 